\title{
REPRESENTATION FUNCTIONS OF SEQUENCES IN ADDITIVE NUMBER THEORY
}

\author{
MELVYN B. NATHANSON ${ }^{1}$
}

\begin{abstract}
Let $\mathcal{Q}$ be a set of nonnegative integers, and let $r_{2}^{\mathscr{Q}}(n)$ denote the number of representations of $n$ in the form $n=a_{i}+a_{j}$ with $a_{i}, a_{j} \in \mathbb{Q}$. The set $\mathbb{Q}$ is periodic if $a \in \mathbb{Q}$ implies $a+m \in \mathbb{Q}$ for some $m>1$ and all $a>N$. It is proved that if $\mathbb{Q}$ is not periodic, then for every set $B \neq Q$ there exist infinitely many $n$ such that $r_{2}^{Q}(n) \neq r_{2}^{\Phi}(n)$. Moreover, all pairs of periodic sets $\mathscr{Q}$ and $\mathscr{B}$ are constructed that satisfy $r_{2}^{\mathscr{Q}}(n)=r_{2}^{\Phi}(n)$ for all but finitely many $n$.
\end{abstract}

Let $\mathcal{Q}$ be a set of nonnegative integers. Let $r_{h}^{\mathbb{Q}}(n)$ denote the number of representations of $n$ as a sum of $h$ elements of $\mathcal{Q}$. If $f_{\mathfrak{Q}}(z)=\Sigma_{a \in \mathbb{Q}^{2}}$ is the generating function for $\mathcal{Q}$, then $f_{\mathbb{Q}}(z)^{h}=\sum_{n=0}^{\infty} r_{h}^{\mathbb{Q}}(n) z^{n}$. Let $r^{\mathbb{Q}}(n)$ denote the number of representations of $n$ as a sum of an arbitrary number of elements of $\mathcal{Q}$. If $0 \notin \mathbb{Q}$, then $r^{\mathbb{Q}}(n)=\sum_{h=1}^{\infty} r_{h}^{\mathbb{Q}}(n)$ is finite for all $n$. Representation functions have been studied by various authors [1]-[7].

In this note I consider the question: To what extent do the sequences $r_{h}^{Q}(n)$ and $r^{\mathbb{Q}}(n)$ determine the set $\mathcal{Q}$ ? I shall prove that if $\mathcal{Q}$ and $\mathscr{B}$ are sets of nonnegative integers such that $r_{h}^{\mathbb{Q}}(n)=r_{h}^{\mathbb{P}}(n)$ for some $h \geqslant 1$ and all $n \geqslant 0$, or if $r^{\mathbb{Q}}(n)=r^{\mathscr{B}}(n)$ for all $n \geqslant 0$, then $\mathbb{Q}=\mathscr{B}$. However, there do exist sets $\mathbb{Q}$ and $\mathscr{B}$ such that $r_{2}^{\mathbb{Q}}(n)=r_{2}^{\mathscr{B}}(n)$ for all sufficiently large $n$, but $\mathbb{Q} \neq \mathscr{B}$. All such pairs of sets $\mathbb{Q}$ and $\mathscr{B}$ will be constructed explicitly. An infinite set $\mathscr{Q}$ of integers is called periodic if there exist integers $m \geqslant 1$ and $N$ such that $a \in \mathbb{Q}$ implies $a+m \in \mathbb{Q}$ for all $a>N$. It will be shown that if the set $\mathbb{Q}$ is not periodic, then for every set $\mathscr{B} \neq \mathbb{Q}$ we must have $r_{2}^{\mathbb{Q}}(n) \neq r_{2}^{\mathscr{B}}(n)$ for infinitely many $n$.

THEOREM 1. Let $\mathcal{Q}$ and $\mathscr{B}$ be sets of nonnegative integers, and let $r_{h}^{\mathscr{Q}}(n)$ and $r_{h}^{\mathbb{B}}(n)$ denote the number of representations of $n$ as a sum of $h$ elements of $\mathbb{Q}$ and $\mathscr{B}$, respectively. If $r_{h}^{\mathbb{Q}}(n)=r_{h}^{\mathscr{B}}(n)$ for all $n \geqslant 0$, then $\mathcal{Q}=\mathscr{B}$.

PRoof. If $r_{h}^{\mathbb{Q}}(n)=r_{h}^{\mathscr{B}}(n)$ for all $n \geqslant 0$, then

$$
\begin{aligned}
0 & =\sum_{n=0}^{\infty} r_{h}^{\mathbb{Q}}(n) z^{n}-\sum_{n=0}^{\infty} r_{h}^{\mathscr{B}}(n) z^{n}=f_{\mathscr{Q}}(z)^{h}-f_{\mathscr{B}}(z)^{h} \\
& =\left(f_{\mathscr{Q}}(z)-f_{\mathscr{B}}(z)\right)\left(f_{\mathscr{Q}}(z)^{h-1}+f_{\mathscr{Q}}(z)^{h-2} f_{\mathscr{B}}(z)+\cdots+f_{\mathscr{B}}(z)^{h-1}\right) .
\end{aligned}
$$

Received by the editors February 14, 1977.

AMS (MOS) subject classifications (1970). Primary 10L15, 10L05, $10 \mathrm{~J} 99$.

Key words and phrases. Representation functions, addition of sequences, sum sets.

'This paper was written in July, 1976, at the Institute for Advanced Study, Princeton, New Jersey. The author wishes to thank the Institute for its hospitality. 
But $f_{\mathscr{Q}}(z)^{h-1}+\cdots+f_{\mathscr{B}}(z)^{h-1}$ is a nonzero power series with nonnegative coefficients, and so $f_{\mathscr{Q}}(z)-f_{\mathscr{B}}(z)=0$, that is, $Q=\mathscr{B}$.

THEOREM 2. Let $\mathcal{Q}$ and $\mathscr{B}$ be sets of positive integers, and let $r^{\mathbb{Q}}(n)$ and $r^{\mathscr{B}}(n)$ denote the number of representations of $n$ as a sum of an arbitrary number of elements of $Q$ and $\mathscr{B}$, respectively. If $r^{\mathbb{Q}}(n)=r^{\mathscr{B}}(n)$ for all $n \geqslant 1$, then $Q=\Re$.

Proof. Let $r^{\mathbb{Q}}(0)=r_{0}^{\mathbb{Q}}(0)=1$ and let $r_{0}^{\mathbb{Q}}(n)=0$ for all $n>1$. Clearly,

$$
r^{\mathfrak{Q}}(n)=\sum_{h=0}^{\infty} r_{h}^{\mathbb{Q}}(n) \text { for all } n \geqslant 0 .
$$

Therefore,

$$
\begin{aligned}
\sum_{n=0}^{\infty} r^{\mathbb{Q}}(n) z^{n} & =\sum_{n=0}^{\infty} \sum_{h=0}^{\infty} r_{h}^{\mathbb{Q}}(n) z^{n}=\sum_{h=0}^{\infty} \sum_{n=0}^{\infty} r_{h}^{\mathbb{Q}}(n) z^{n} \\
& =\sum_{h=0}^{\infty} f_{\mathscr{Q}}(z)^{h}=\frac{1}{1-f_{\mathbb{Q}}(z)}
\end{aligned}
$$

Consequently, if $r^{\mathscr{Q}}(n)=r^{\mathscr{B}}(n)$ for all $n \geqslant 0$, then $\left(1-f_{\mathscr{d}}(z)\right)^{-1}=(1-$ $\left.f_{\mathscr{B}}(z)\right)^{-1}$, and so $\mathcal{Q}=\mathscr{B}$.

THEOREM 3. Let $\mathcal{Q}$ and $\mathscr{B}$ be sets of positive integers, and let $p^{\mathscr{Q}}(n)$ and $p^{\mathscr{B}}(n)$ denote the number of representations of $n$ as the sum of an arbitrary number of elements of $\mathcal{Q}$ and $\mathscr{B}$, respectively, where representations differing only in the arrangement of their summands are not counted separately. If $p^{\mathbb{Q}}(n)=p^{\mathscr{B}}(n)$ for all $n \geqslant 1$, then $\mathbb{Q}=\mathscr{B}$.

Proof. Let $p^{\mathscr{Q}}(0)=p^{\mathscr{B}}(0)=1$. The generating function for $p^{\mathscr{Q}}(n)$ has the form

$$
\sum_{n=0}^{\infty} p^{\mathscr{Q}}(n) z^{n}=\prod_{a \in \mathcal{Q}} \sum_{k=0}^{\infty} z^{a k}=\prod_{a \in \mathbb{Q}} \frac{1}{1-z^{a}}
$$

If $p^{\mathscr{Q}}(n)=p^{\mathscr{B}}(n)$ for all $n \geqslant 1$, then $\Pi_{a \in \mathbb{Q}}\left(1-z^{a}\right)=\Pi_{b \in \mathscr{B}}\left(1-z^{b}\right)$, and so $Q=\Re$.

If $Q$ and $\mathscr{B}$ are sets of nonnegative integers such that $r_{h}^{\mathscr{Q}}(n)=r_{h}^{\mathscr{B}}(n)$ for some $h \geqslant 1$ and all sufficiently large $n$, then it does not follow that $Q=\mathscr{B}$. For example, $r_{1}^{\mathbb{Q}}(n)=1$ if $n \in \mathbb{Q}$ and $r_{1}^{\mathbb{Q}}(n)=0$ if $n \notin \mathbb{Q}$, and so $r_{1}^{\mathbb{Q}}(n)=$ $r_{1}^{\mathscr{B}}(n)$ for all sufficiently large $n$ if and only if $\mathscr{Q}$ and $\mathscr{B}$ eventually coincide. If $Q=\{n \geqslant 1\}$ and $\mathscr{B}=\{0\} \cup\{n \geqslant 2\}$, then $r_{2}^{\mathscr{Q}}(n)=r_{2}^{\mathscr{B}}(n)$ for all $n \geqslant 3$, but $\mathbb{Q} \neq \mathscr{B}$. This construction can be generalized in the following way.

Let $A, B$, and $T$ be finite sets of integers. If each residue class modulo $m$ contains exactly the same number of elements of $A$ as elements of $B$, then we write $A \equiv B(\bmod m)$. If the number of solutions of the congruence $a+t \equiv$ 
$n(\bmod m)$ with $a \in A, t \in T$, equals the number of solutions of the congruence $b+t \equiv n(\bmod m)$ with $b \in B, t \in T$, for each residue class $n$ modulo $m$, then we write $A+T \equiv B+T(\bmod m)$.

Let $A, B$, and $T$ be finite sets of integers with $A \cup B \subset\{0,1,2, \ldots, N\}$ and $T \subset\{0,1, \ldots, m-1\}$ such that $A+T \equiv B+T(\bmod m)$ for some $m>1$. Define the periodic set $C$ by

$$
\mathcal{C}=\{c>N \mid c \equiv t(\bmod m) \text { for some } t \in T\} \text {. }
$$

Let $\mathbb{Q}=A \cup \mathcal{C}$ and $\mathscr{B}=B \cup \mathcal{C}$. If $A \neq B$, then $Q \neq \mathscr{B}$. We shall prove that $r_{2}^{\mathbb{Q}}(n)=r_{2}^{\mathscr{B}}(n)$ for all $n>2 N$.

Let $s^{A}(n)$ (resp. $s^{B}(n)$ ) denote the number of representations of $n$ in the form $n=a+c$ with $a \in A, c \in \mathcal{C}$ (resp. $n=b+c$ with $b \in B, c \in \mathcal{C}$ ). If $a_{1}, a_{2} \in A$, then $a_{1} \leqslant N$ and $a_{2} \leqslant N$, hence $a_{1}+a_{2} \leqslant 2 N$. Consequently, if $n>2 N$ then the only representations of $n$ as a sum of two elements of $Q=A \cup \mathcal{C}$ are of the form $a+c, c+a$, and $c+c^{\prime}$, where $a \in A$ and $c, c^{\prime} \in \mathcal{C}$. Since $A \cap \mathcal{C}=\varnothing$, this implies that $r_{2}^{\mathbb{Q}}(n)=r_{2}^{\mathcal{C}}(n)+2 s^{A}(n)$. Similarly, $r_{2}^{\mathscr{B}}(n)=r_{2}^{\mathcal{C}}(n)+2 s^{B}(n)$.

Therefore, $r_{2}^{\mathbb{Q}}(n)=r_{2}^{\mathscr{B}}(n)$ if and only if $s^{A}(n)=s^{B}(n)$.

But if $n>2 N$, then $s^{A}(n)$ is exactly the number of solutions of the congruence $n \equiv a+t(\bmod m)$ with $a \in A, t \in T$. For if $n \equiv a+t$ $(\bmod m)$, then $n-a \equiv t(\bmod m)$ and $n-a>2 N-a \geqslant N$, hence $n-a$ $=c \in \mathcal{C}$, and $n=a+(n-a)=a+c$. Conversely, if $n=a+c$ with $a \in$ $A, c \in \mathcal{C}$, then $c=n-a>2 N-a \geqslant N$, and so $c \equiv t(\bmod m)$ for some $t \in T$, and $n \equiv a+t(\bmod m)$.

Similarly, $s^{B}(n)$ is the number of solutions of the congruence $n \equiv b+t$ $(\bmod m)$ with $b \in B, t \in T$. Since $A+T \equiv B+T(\bmod m)$, this implies that $s^{A}(n)=s^{B}(n)$, and so $r_{2}^{\mathbb{Q}}(n)=r_{2}^{\mathscr{B}}(n)$ for all $n>2 N$. The following theorem shows that this construction produces all pairs of sets $\mathbb{Q}$ and $\mathscr{B}$ such that $r_{2}^{\mathbb{Q}}(n)=r_{2}^{\mathscr{B}}(n)$ for all sufficiently large $n$.

THEOREM 4. Let $\mathbb{Q}$ and $\mathscr{B}$ be sets of nonnegative integers such that $r_{2}^{\mathbb{Q}}(n)=$ $r_{2}^{G}(n)$ for all sufficiently large $n$. Then there exist finite sets $A, B$, and $T$ with $A \cup B \subset\{0,1, \ldots, N\}$ and $T \subset\{0,1, \ldots, m-1\}$ such that $A+T \equiv B$ $+T(\bmod m)$, and $Q=A \cup \mathcal{C}$ and $\mathscr{B}=B \cup \mathcal{C}$, where $\mathcal{C}=\{c>N \mid c \equiv t$ $(\bmod m)$ for some $t \in T\}$.

Proof. To every representation $2 n=a_{i}+a_{j}$ with $a_{i}, a_{j} \in \mathbb{Q}$ and $a_{i} \neq a_{j}$ corresponds a second representation $2 n=a_{j}+a_{i}$. Therefore, $r_{2}^{\mathbb{Q}}(2 n)$ is odd if and only if $n \in \mathbb{Q}$. Similarly, $n \in \mathscr{B}$ if and only if $r_{2}^{\mathscr{B}}(n)$ is odd. If $r_{2}^{\mathbb{Q}}(n)=$ $r_{2}^{B}(n)$ for all $n>N_{0}$, then for all $n>N_{0}$ we have $n \in \mathbb{Q}$ if and only if $n \in \mathscr{B}$. Let $f_{\mathscr{Q}}(z)=\sum_{a \in \mathbb{Q}^{z}}{ }^{a}$ and $f_{\mathscr{B}}(z)=\sum_{b \in \mathscr{B}} z^{b}$. Then $f_{\mathscr{Q}}(z)-f_{\mathscr{B}}(z)=Q(z)$ is a polynomial of degree at most $N_{0}$, and $f_{\mathscr{Q}}(z)+f_{\mathscr{B}}(z)$ is a power series such that, for $n>N_{0}$, the coefficient of $z^{n}$ is either 0 (if $n \notin \mathbb{Q} \cup \mathscr{B}$ ) or 2 (if $n \in \mathbb{Q} \cap \mathscr{B})$. Then 


$$
\begin{aligned}
Q(z)\left(f_{\mathscr{Q}}(z)+f_{\mathscr{B}}(z)\right) & =\left(f_{\mathfrak{Q}}(z)-f_{\mathscr{B}}(z)\right)\left(f_{\mathscr{Q}}(z)+f_{\mathscr{B}}(z)\right) \\
& =f_{\mathscr{Q}}(z)^{2}-f_{\mathscr{B}}(z)^{2}=\sum_{n=0}^{\infty}\left(r_{2}^{\mathbb{Q}}(n)-r_{2}^{\mathscr{B}}(n)\right) z^{n} \\
& =\sum_{n=0}^{N_{0}}\left(r_{2}^{\mathbb{Q}}(n)-r_{2}^{\mathscr{B}}(n)\right) z^{n}=P(z),
\end{aligned}
$$

where $P(z)$ is a polynomial of degree at most $N_{0}$. Therefore, $f_{\mathscr{Q}}(z)+f_{\mathscr{B}}(z)=$ $P(z) / Q(z)$ is a rational function, and so the sequence of coefficients of the power series $f_{\mathscr{Q}}(z)+f_{\mathscr{B}}(z)$ eventually satisfies a linear recurrence relation. These coefficients are eventually either 0 or 2 . But a sequence defined by a linear recurrence on a finite set must be eventually periodic. This implies that for some modulus $m \geqslant 1$ there exists a set $T \subset\{0,1,2, \ldots, m-1\}$ and an integer $N>N_{0}$ such that, for all $n>N$, we have $n \in \mathbb{Q} \cap \mathscr{B}$ if and only if $n \equiv t(\bmod m)$ for some $t \in T$.

Let

$$
A=\{a \leqslant N \mid a \in \mathbb{Q}\}, \quad B=\{b \leqslant N \mid b \in \mathscr{B}\},
$$

and

$$
\mathcal{C}=\{c>N \mid c \in \mathbb{Q} \cap \mathscr{B}\}=\{c>N \mid c \equiv t(\bmod m) \text { for some } t \in T\} .
$$

Then $\mathbb{Q}=A \cup \mathcal{C}$ and $\mathscr{B}=B \cup \mathcal{C}$.

If $n>2 N$, then $r_{2}^{\mathbb{Q}}(n)=r_{2}^{\mathcal{E}}(n)+2 s^{A}(n)$ and $r_{2}^{\mathscr{B}}(n)=r_{2}^{\mathcal{C}}(n)+2 s^{B}(n)$, where $s^{A}(n)$ (resp. $s^{B}(n)$ ) is the number of representations of $n$ in the form $n=a+c$ with $a \in A, c \in \mathcal{C}$ (resp. $n=b+c$ with $b \in B, c \in \mathcal{C}$ ). Since $r_{2}^{\mathbb{Q}}(n)=r_{2}^{B}(n)$ for $n>2 N$, it follows that $s^{A}(n)=s^{B}(n)$. But $s^{A}(n)$ (resp. $\left.s^{B}(n)\right)$ is also the number of solutions of the congruence $n \equiv a+t(\bmod m)$ with $a \in A, t \in T($ resp. $n \equiv b+t(\bmod m)$ with $b \in B, t \in T)$. Therefore, $A+T \equiv B+T(\bmod m)$.

COROLlaRY. If the set $\mathbb{Q}$ of nonnegative integers is not eventually periodic, then for every set $\mathscr{B} \neq \mathbb{Q}$ we have $r_{2}^{\mathbb{Q}}(n) \neq r_{2}^{\mathscr{B}}(n)$ for infinitely many $n$.

REMARKS. The converse of this corollary is false. If $\mathcal{Q}$ is the sequence of all nonnegative integers, then $r_{2}^{\mathbb{Q}}(n)=n+1$ for all $n \geqslant 0$. If $\mathscr{B} \neq \mathbb{Q}$, then $r_{2}^{B}(n) \leqslant n$ for all sufficiently large $n$. But the sequence $Q$ is periodic with period 1 .

It is an open problem to determine those sets $Q$ and $\mathscr{B}$ such that $r_{h}^{\mathbb{Q}}(n)=r_{h}^{\Phi_{B}}(n)$ for some $h \geqslant 3$ and all sufficiently large $n$.

The following problem arises from Theorem 4 . Let $A, B$, and $T$ be finite nonempty sets of nonnegative integers with $T \subset\{0,1,2, \ldots, m-1\}$ for some $m \geqslant 1$ such that $A+T \equiv B+T(\bmod m)$. Assume the modulus $m$ is "reduced" in the sense that there does not exist a divisor $m_{1}$ of $m$ and a set $T_{1} \subset\left\{0,1, \ldots, m_{1}-1\right\}$ such that $T=\left\{t \in\{0,1, \ldots, m-1\} \mid t \equiv t_{1}\right.$ $\left(\bmod m_{1}\right)$ for some $\left.t_{1} \in T_{1}\right\}$. Does this imply that $A \equiv B(\bmod m)$ ? This is 
obviously true if $m=1$. The following result proves this is also true if the modulus $m$ is prime.

THEOREM 5. Let $p$ be a prime number, and let $A, B, T$ be finite, nonempty sets of nonnegative integers with $T \subset\{0,1, \ldots, p-1\}$. If $T=\{0,1, \ldots, p-$ 1\}, then $A+T \equiv B+T(\bmod p)$ if and only if $|A|=|B|$. If $T \subset$ $\{0,1, \ldots, p-1\}$, then $A+T \equiv B+T(\bmod p)$ if and only if $A \equiv{ }_{B}$ $(\bmod p)$.

Proof. If $\mathrm{T}=\{0,1, \ldots, p-1\}$, then the number of solutions of the congruence $a+t \equiv n(\bmod p)$ with $a \in A, t \in T(\operatorname{resp} . b+t \equiv n(\bmod p)$ with $b \in B, t \in T$ ) is exactly $|A|$ (resp. $|B|$ ) for all $n$. Therefore, $A+T \equiv B$ $+T(\bmod p)$ if and only if $|A|=|B|$.

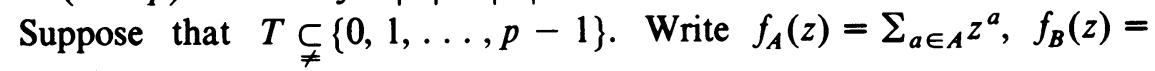
$\sum_{b \in B} z^{b}$, and $f_{T}(z)=\sum_{t \in T^{z}}$. If $A+T \equiv B+T(\bmod p)$, then

$$
f_{A}(z) f_{T}(z) \equiv f_{B}(z) f_{T}(z) \quad\left(\bmod z^{p}-1\right)
$$

that is, $z^{p}-1$ divides $\left(f_{A}(z)-f_{B}(z)\right) f_{T}(z)$. The cyclotomic polynomial $z^{p}-$ 1 is the product of two irreducible factors

$$
z^{p}-1=(z-1)\left(1+z+z^{2}+\cdots+z^{p-1}\right) .
$$

The degree of $f_{T}(z)$ is at most $p-1$, and so, if $1+z+\cdots+z^{p-1}$ divides $f_{T}(z)$, then $f_{T}(z)=1+z+\cdots+z^{p-1}$ and $T=\{0,1, \ldots, p-1\}$, which is false. Therefore, $1+z+\cdots+z^{p-1}$ divides $f_{A}(z)-f_{B}(z)$. If $z-1$ divides $f_{T}(z)$, then $f_{T}(z)=(z-1) g(z)$ for some polynomial $g(z)$. But this implies that $f_{T}(1)=0$. However, since $f_{T}(z)=\Sigma_{t \in T^{t}}$, it follows that $f_{T}(1)=$ $|T| \neq 0$ since $T \neq \varnothing$. Therefore, $z-1$ also divides $f_{A}(z)-f_{B}(z)$. Consequently, $z^{p}-1$ divides $f_{A}(z)-f_{B}(z)$. But this means precisely that $A \equiv B$ $(\bmod p)$.

COROllary. Let $p$ be a prime, and let $A, B$, and $T$ be nonempty, proper subsets of $\{0,1, \ldots, p-1\}$. If $A+T \equiv B+T(\bmod p)$, then $A=B$.

\section{REFERENCES}

1. A. O. L. Atkin, On pseudo-squares, Proc. London Math. Soc. (3) 14 (1965), 22-27.

2. P. T. Bateman, E. E. Kohlbecker and J. P. Tull, On a theorem of Erdis and Fuchs in additive number theory, Proc. Amer. Math. Soc. 14 (1963), 278-284.

3. P. Erdös and W. H. J. Fuchs, On a problem of additive number theory, J. London Math. Soc. 31 (1956), 67-73.

4. P. Erdøs, B. Gordon, L. A. Rubel and E. G. Straus, Tauberian theorems for sum sets, Acta Arith. 9 (1964), $177-189$.

5. P. Erdös and A. Rényi, Additive properties of random sequences of positive integers, Acta Arith. 6 (1960), 83-110.

6. P. Erdös and P. Turán, On a problem of Sidon in additive number theory, and on some related problems, J. London Math. Soc. 16 (1941), 212-215.

7. R. C. Vaughan, On the addition of sequences of integers, J. Number Theory 4 (1972), 1-16.

Department of Mathematics, SOUthern Illinois University, Carbondale, Illinois 62901 